\title{
Affimer-Enzyme-Inhibitor Switch Sensor for Rapid Wash-free Assays of Multimeric Proteins
}

\author{
Hope Adamson, ${ }^{\dagger, \ddagger 0}$ Modupe O. Ajayi, ${ }^{\ddagger}, \S$ Emma Campbell, ${ }^{\dagger, \ddagger}$ Erika Brachi, ${ }^{\dagger, \ddagger}$ Christian Tiede, ${ }^{\ddagger}, \S$ \\ Anna A. Tang,

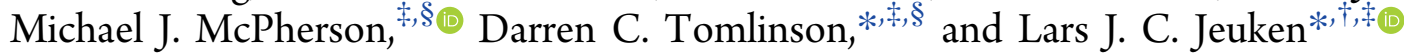 \\ ${ }^{\dagger}$ School of Biomedical Sciences, ${ }^{\ddagger}$ Astbury Centre for Structural Molecular Biology, and ${ }^{\S}$ School of Molecular and Cellular Biology, \\ University of Leeds, Leeds LS2 9JT, U.K. \\ ${ }$ Avacta Life Sciences Limited, Unit 20, Ash Way, Thorp Arch Estate, Wetherby LS23 7FA, U.K.
}

\section{Supporting Information}

\begin{abstract}
Robust technology is required to underpin rapid point-of-care and in-field diagnostics to improve timely decision making across broad sectors. An attractive strategy combines target recognition and signal generating elements into an "active" enzyme-switch that directly transduces targetbinding into a signal. However, approaches that are broadly applicable to diverse targets remain elusive. Here, an enzymeinhibitor switch sensor was developed by insertion of non-

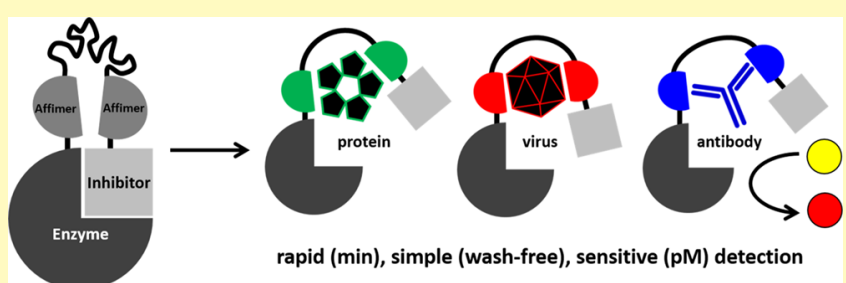
immunoglobulin Affimer binding proteins, between TEM1- $\beta$-lactamase and its inhibitor protein, such that target binding disrupts the enzyme-inhibitor complex. Design principles for a successful switch architecture are illustrated by the rapid (min), simple (wash-free), and sensitive ( $\mathrm{pM}$ ) quantification of multimeric target analytes in biological samples (serum, plasma, leaf extracts), across three application areas. A therapeutic antibody (Herceptin), protein biomarker (human C-reactive protein), and plant virus (cow pea mosaic virus) were targeted, demonstrating assays for therapeutic drug monitoring, health diagnostics, and plant pathogen detection, respectively. Batch-to-batch reproducibility, shelf-life stability, and consistency with validated enzyme-linked immunosorbent assay analysis confirm that the principle of an Affimer-enzyme-inhibitor switch provides a platform for point-of-care and in-field diagnostics.
\end{abstract}

KEYWORDS: protein switch, homogenous assay, wash-free diagnostics, protein engineering, Affimers, point-of-care, in-field, biosensor

A general assay platform for rapid detection of diverse analytes is needed to underpin development of new point-of-care and in-field diagnostics. Such tests are vital to accelerate and improve decision making relative to laboratory assays, across broad sectors including medicine, environmental monitoring, and agricultural management. ${ }^{1-4}$ The enzymelinked immunosorbent assay (ELISA) has long been the gold standard of immunoassays, as it is a highly sensitive and modular format. ${ }^{5,6}$ However, the use of separate molecular recognition "capture" reagents (e.g., antibodies) and signal generating "detection" reagents (e.g., antibody-enzyme conjugates) necessitates the use of multiple time-consuming immobilisation and wash steps, precluding use for point-of-care diagnostics. $^{7}$ Engineered solutions include lateral flow and microfluidic systems with automated steps, but these are often semi-quantitative and can lack sensitivity. ${ }^{8,9}$ To overcome these limitations, the "capture" and "detection" elements can be combined at the molecular level to create an "active" enzyme-switch sensor, which directly transduces target binding into a signal. ${ }^{7,10}$

Devising an efficient enzyme switching mechanism that functions for diverse targets remains a significant challenge. Approaches based on split enzyme complementation can suffer from low in vitro stability and limited activity recovery. ${ }^{11-13}$ Target-driven modulation of bioluminescent resonant energy transfer (BRET) has been predominantly used for small molecule and antibody sensing. ${ }^{14-20}$ Synthetic allosteric switches often give subtle, unpredictable signal changes, and so are hard to generalize for varied targets. ${ }^{10,21,22}$ A recent twocomponent system offers a solution, whereby a consistent peptide and peptide-responsive allosteric switch are brought together in the presence of target by exchangeable binding proteins. ${ }^{23}$ However, because of self-association, two-component systems are inherently highly dependent on sensor component concentrations, which may limit performance and sensitivity. ${ }^{24,25}$ Single-protein switches avoid this and further allow for avidity effects that enhance selectivity and sensitivity, and so could prove more robust. ${ }^{24,26}$

Target-driven disruption of a linked enzyme-inhibitor complex is a promising strategy. Two recognition elements are incorporated such that target binding by both leads to conformational disruption of the enzyme-inhibitor interaction

Received: August 15, 2019

Accepted: October 3, 2019

Published: October 3, 2019 
(A) Construct BB_HA
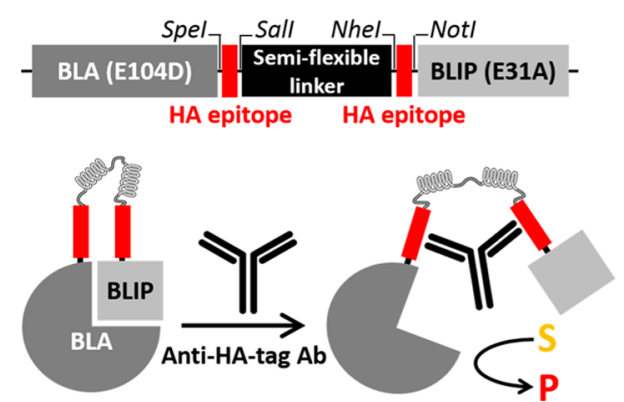

(B) General construct BLA-L1-AAff-L2-BAff-L3-BLIP

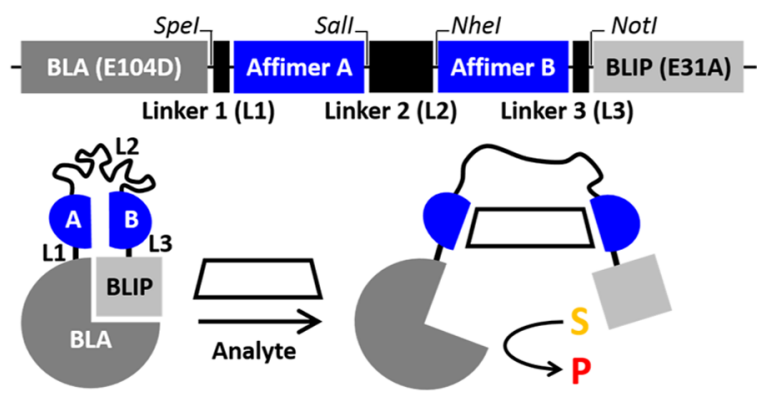

Figure 1. Construct designs and enzyme switch mechanisms for (A) BB_HA, anti-HA-tag antibody sensor based on the design described by Banala

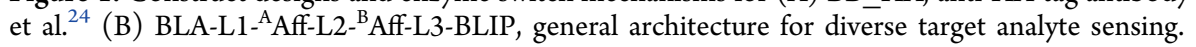

and "switches on" the enzyme. The approach has been exemplified using protease and $\beta$-lactamase reporters for peptide, antibody, and protease detection but, for each, the system is target class-specific and a system that allows broader protein detection remains elusive. ${ }^{24,27-30}$ Here, we introduce an enzyme-inhibitor switch architecture that can be used to detect different multimeric protein analytes. The sensor structure is developed, such that the switching mechanism is functional for three different multimeric targets, via simple exchange of recognition elements. We utilize Affimer affinity reagents, a class of non-immunoglobulin binding protein, based on a cystatin scaffold with two variable target binding regions. $^{31-33}$

They are efficiently selected against diverse targets by phage display to provide high affinity binders with exquisite specificity, enabling timely development of new sensors. ${ }^{32-34}$ With over 500 successful screens to date and uses across wideranging assays, Affimer proteins offer the necessary generality for target detection. ${ }^{32,35-43}$

The applicability of our engineered enzyme-switch sensor is illustrated by detection of different targets in three application areas, including those of unmet need for rapid diagnostics. A therapeutic antibody (Herceptin), pentameric protein biomarker (human C-reactive protein, hCRP), and icosahedral plant virus (cow pea mosaic virus, CPMV) are successfully targeted, providing assays for therapeutic drug monitoring, health diagnostics, and plant pathogen detection, respectively. ${ }^{2,4,44-47}$ Applicability to rapid diagnostics is confirmed by the rapid ( $\mathrm{min}$ ), simple (wash-free), and sensitive (pM) quantification of analytes in biological samples, along with consistency with validated ELISA analysis, robust batch-tobatch reproducibility and stability at fridge and room temperatures.

\section{RESULTS AND DISCUSSION}

Enzyme Switch Design. An antibody-sensing enzymeinhibitor switch described by Banala et al. provided a promising starting point for design of a more general protein sensor. $^{24}$ TEM1- $\beta$-lactamase (BLA) was tethered to its inhibitor protein (BLIP) via a semi-flexible (SF) linker of sequence (GSG) ${ }_{6} \mathrm{~A}(\text { EAAAK })_{6} \mathrm{~A}(\mathrm{GSG})_{6} \mathrm{~A}(\mathrm{EAAAK})_{6} \mathrm{~A}(\mathrm{GSG})_{6}$, known to span the distance between the antigen binding sites on each antibody arm. ${ }^{24}$ Point mutations at the interface of BLA (E104D) and BLIP (E31A) weakened the interaction to ensure efficient dissociation upon binding of an antibody to both epitopes, in this case HA peptides that flank the linker. ${ }^{24}$ BLA uses simple chromogenic and fluorogenic substrates and has relatively fast turnover, enhancing signal amplification and thus sensitivity. ${ }^{24,29}$ This design enabled selective and sensitive detection of multiple antibodies but relied on knowledge of binding epitopes and was unsuitable for wider protein detection. $^{24}$ We introduced restriction sites to enable simple sensor reconfiguration, and this modified BLA-BLIP anti-HAtag antibody sensor (BB_HA) was our initial construct from which a more general protein sensor was engineered (Figure 1A).

The epitopes were exchanged for selectable Affimer coding regions to broaden the target scope (Figure 1B). A minimal Affimer sequence with no additional $\mathrm{N}$ - or C-terminal extensions was used, so that the linker regions predictably define the distance and structure between BLA, the Affimers, and BLIP. These linkers (L1, L2, and L3) are tuneable to account for analytes of varied size and geometry and ensure efficient target binding and BLA-BLIP dissociation. This gives a general construct architecture (Figure 1B), with individual constructs named according to BLA-L1- ${ }^{\mathrm{A}}$ Aff-L2- ${ }^{\mathrm{B}}$ Aff-L3-BLIP where L1, L2, and L3 are the linkers and Aff denotes the Affimer used. We surmised that an optimal underlying sensor architecture could be attained, for which new analytes are successfully targeted by simple Affimer exchange and minimal further optimization.

Affimer Selection. The Affimers must work as a pair, such that Affimers $A$ and $B$ in the enzyme-switch bind two regions of the analyte, to induce BLA-BLIP dissociation. The target analytes are all multimeric and have repeated binding sites, so one Affimer was selected for each and used at both sites in the enzyme switch.

Herceptin Analyte. We have previously identified an antiHerceptin Affimer (Aff-Her) that is highly selective for the two identical Herceptin variable regions on each antibody arm; AffHer is thus used at both Affimer sites in the sensor. ${ }^{48}$

$h C R P$ Analyte. An Affimer phage display library ${ }^{33}$ was screened against biotinylated hCRP with three rounds of panning. A primary screen of outputs from panning rounds 2 and 3 was performed using the iQue Screener (IntelliCyt) and then positive clones were identified by ELISA analysis. Binding of four anti-hCRP Affimers $(25,27,81$ and 90) to hCRP was confirmed with a direct ELISA (Figure S1A). A sandwich ELISA compared the 16 combinations of "capture" and "detection" Affimer (Figure S1B) and Affimer 90 with 90 was the best pair. The same Affimer can bind at two sites on hCRP as it is a pentameric protein with five identical monomers, so anti-hCRP Affimer 90 (Aff-hCRP90) was paired with itself in the sensor construct. 
CPMV Analyte. We have previously identified the highly specific anti-CPMV Affimer 3 (Aff-CPMV3) and used it as both "capture" and "detection" Affimer in a sandwich ELISA. ${ }^{47}$ The icosahedral virus has 60 copies of the large and small subunits and a cryo-EM structure confirmed Affimer binding at 20 equivalent sites ca. $80 \AA$ apart; ${ }^{47}$ Aff-CPMV3 was thus used at both Affimer sites in the sensor.

Each Affimer has nanomolar affinity for its target analyte; the $K_{\mathrm{d}}$ of each was measured by surface plasmon resonance (SPR, Aff-Her, $27 \pm 6 \mathrm{nM}$; Aff-hCRP90, $12 \pm 1 \mathrm{nM}$; Aff-CPMV3, 14 $\pm 6 \mathrm{nM})$.

Example 1: Detection of a Therapeutic Antibody (Herceptin). Herceptin (trastuzumab) is a therapeutic monoclonal antibody ( $\mathrm{mAb})$ used to treat breast cancer, but as with many mAbs, there is significant interpatient variability in drug clearance rates. ${ }^{44,45,49}$ Thus, there is a need for pointof-care diagnostics to enable therapeutic drug monitoring for personalized dosing, which would improve the efficacy of Herceptin and wider $\mathrm{mAb}$ therapy. ${ }^{45}$ Monomeric antibodies share a common structure that presents two antigen binding sites ca. $100 \AA$ apart and the SF linker between epitopes in the anti-HA-tag antibody sensor (BB_HA) is known to efficiently span this gap. ${ }^{24}$ We therefore surmised that simple exchange of the HA-tag epitopes in BB_HA for anti-Herceptin Affimers (Aff-Her) would generate a functional Herceptin sensor (BB_Her). BB_HA and BB_Her were purified using first an $\mathrm{N}$-terminal His-tag and then a C-terminal Strep-tag to give pure full-length sensor proteins (Figure S2).

The dose responses of BB_HA and BB_Her to anti-HA-tag antibody and Herceptin, respectively, were assessed using a simple colorimetric homogenous assay (Figure 2). The sensor $(2 \mathrm{nM})$ and varying concentrations of analyte were incubated for $15 \mathrm{~min}$ prior to addition of $50 \mu \mathrm{M}$ nitrocefin substrate, in a $200 \mu \mathrm{L}$ well. In the presence of active $\beta$-lactamase, yellow nitrocefin is hydrolyzed to a red product, which was monitored by absorbance at $551 \mathrm{~nm}\left(A_{551}\right), 15 \mathrm{~min}$ after nitrocefin addition. BB_Her, like BB_HA, was activated by its target

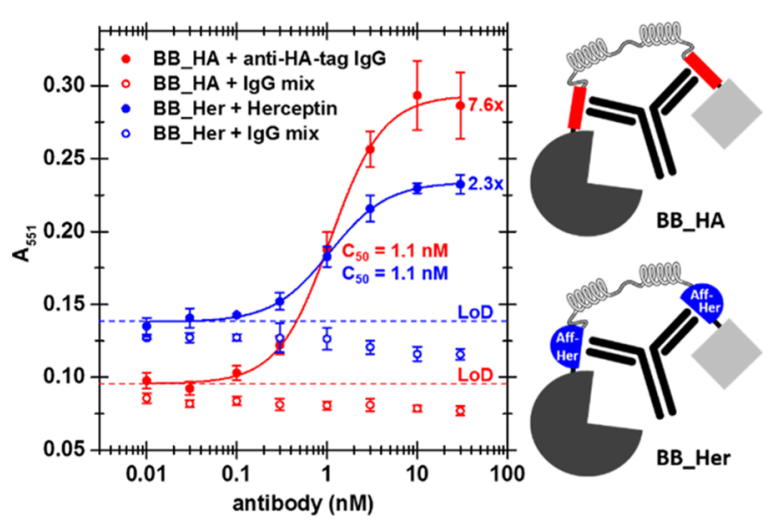

Figure 2. Dose response curves of BB_HA and BB_Her to anti-HAtag IgG, Herceptin, and a non-specific human IgG mix in a colorimetric homogenous assay. The sensor $(2 \mathrm{nM})$ was incubated with varying antibody concentrations for $15 \mathrm{~min}$ before addition of 50 $\mu \mathrm{M}$ nitrocefin. Absorbance was read at $551 \mathrm{nM}, 15 \mathrm{~min}$ after nitrocefin addition. Data points are the mean of three independent measurements and error bars indicate standard deviation from the mean. Solid lines are $4 \mathrm{PL}$ regression fits and $\mathrm{C}_{50}$ (antibody concentration for $50 \%$ of the maximum sensor response) values are indicated. Dash lines indicate the limit of detection (LoD), and fold activity gains $\Delta A_{551}(30 \mathrm{nM} \mathrm{Ab}) / \Delta A_{551}(0 \mathrm{nM} \mathrm{Ab})$ are indicated. antibody resulting in an increase in $A_{551}$ with target concentration; at saturating antibody concentrations, BB_HA and BB_Her displayed 7.6- and 2.3-fold activity gains, respectively, relative to their background activity with no antibody. Both dose response curves can be fit to a four parameter logistic (4PL) regression with $C_{50}=1.1 \mathrm{nM}$. As with BB_HA, BB_Her is unresponsive to a mix of human IgG, confirming selectivity for the target antibody. The LoD was calculated as outlined by Armbruster and Pry; ${ }^{50}$ LoD = mean $_{\text {blank }}+1.645\left(\mathrm{SD}_{\text {blank }}\right)+1.645\left(\mathrm{SD}_{\text {low conc. test }}\right)$. Unlike the often used mean blank $+3\left(\mathrm{SD}_{\text {blank }}\right)$ method, these stricter definitions account for variability in both test and blank measurements, including those with non-specific IgG. Instead of an extrapolated LoD concentration, the lowest test concentration explicitly measured above the LoD is taken, giving LoD $=100 \mathrm{pM}$ for both BB_HA and BB_Her. The similar functionality and sensitivity of $\mathrm{BB}$ Her compared to BB_HA under these conditions indicates that BLA, the Affimer proteins, BLIP, and the underlying switch mechanism all remain functional in the new multidomain structure.

The clinically relevant concentration range of Herceptin in serum is ca. 70-2000 nM, which could be measured by BB Her with ca. 250-fold dilution of serum. ${ }^{49,51}$ Together with the speed (30 min) and simplicity (wash-free, simple read out) of the assay, the enzyme-switch holds promise for point-of-care therapeutic drug monitoring for Herceptin and other biotherapeutics. Affimer proteins that bind mAbs are easily developed with high selectivity, ${ }^{48}$ increasing the scope and speed of antibody sensor development compared to using known epitopes or performing time-consuming epitope mapping. Furthermore, BB Her offers improved sensitivity over a BRET enzyme-switch sensor for trastuzumab $(\text { Herceptin })^{52}$ and recently described DNA-switch antibody sensors. ${ }^{53}$

Example 2: Detection of Protein Biomarker (hCRP). The use of Affimer proteins rather than epitopes in the enzyme-switch design provides the opportunity to measure a broad range of analytes, other than antibodies. The core structure of BB_HA and BB_Her was, however, designed for antibody detection, so a step-wise engineering strategy was undertaken to design an enzyme-switch architecture capable of more general multimeric protein detection. The initial protein target was hCRP, an acute-phase inflammatory biomarker, used in the diagnosis and monitoring of numerous diseases, from infectious to rheumatologic and cardiovascular. ${ }^{46,54}$ Point-of-care hCRP tests are currently used to aid diagnosis of viral versus bacterial infections for antimicrobial stewardship, but higher sensitivity tests are needed for cardiovascular disease (CVD) risk monitoring. ${ }^{55,56}$ The anti-hCRP Affimer, Aff-hCRP90 (90), was introduced at both sites in the enzyme switch and the SF linker $2(\mathrm{~L} 2=\mathrm{SF})$ maintained, as the pentameric structure of hCRP suggests that Affimer binding sites should be no more than $100 \AA$ apart. ${ }^{57}$ The purpose of linker $1(\mathrm{~L} 1)$ and linker $3(\mathrm{~L} 3)$ is to translate Affimer movement into BLA-BLIP disruption. They must be sufficiently long to enable efficient Affimer-target binding (without steric effects) but short or rigid enough to ensure BLA-BLIP dissociation, even if the Affimer proteins bind at closer sites than in antibodies $(<100 \AA)$. L1 and L3 were tested with between three and six amino acids (BLA$\left.\mathrm{L1}_{3-6}{ }^{\mathrm{A}} 90-{ }^{\mathrm{L} 2} \mathrm{SF}-{ }^{\mathrm{B}} 90-\mathrm{L} 3_{3-6}-\mathrm{BLIP}\right)$. The sensor with the shortest linkers $(\mathrm{L} 1=\mathrm{TSA}, \mathrm{L} 3=\mathrm{AAA})$ had the greatest hCRP-induced activity gain, but even with $10 \mathrm{nM}$ hCRP, this was only 1.8 -fold 
(A) Varied L1 and L3 with $L 2=$ semi-flexible (SF) (BLA-L1-A90-'²SF-B90-L3-BLIP)

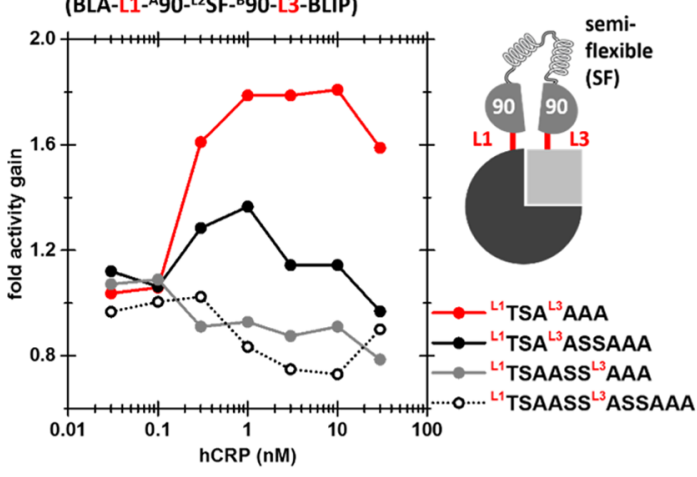

(C) Varied L1 with $L 2=(G S G)_{7}$ and $L 3=A A A$

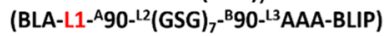

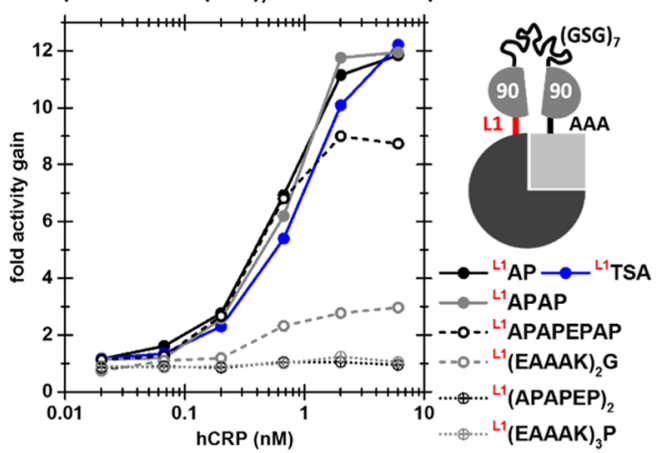

(B) Varied L2 with L1 = TSA and L3 = AAA (BLA- ${ }^{11}$ TSA-A $90-$-L2- ${ }^{B} 90-{ }^{13}$ AAA-BLIP)

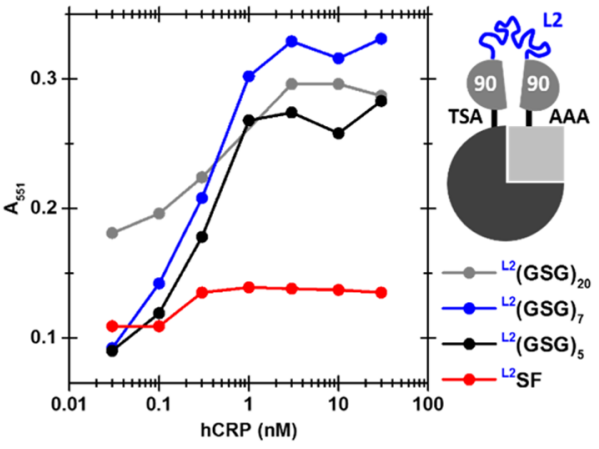

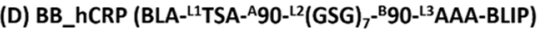
(and controls)

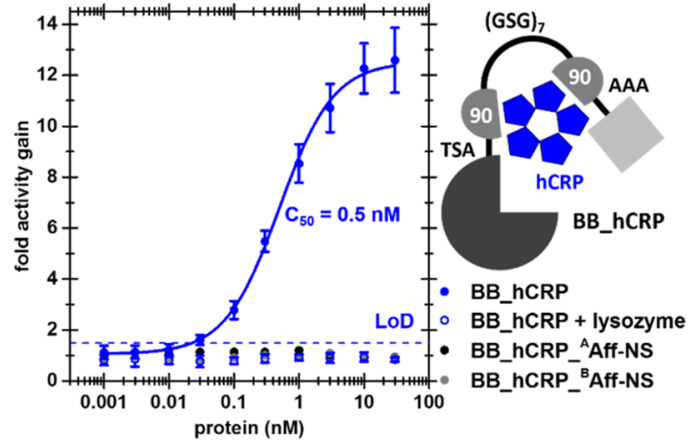

Figure 3. Re-engineering the enzyme-switch for protein (hCRP) detection. (A) Optimizing L1 and L3 (with SF L2 = (GSG) $\mathrm{A}(\mathrm{EAAAK})_{6} \mathrm{~A}$ $\left.(\mathrm{GSG})_{6} \mathrm{~A}(\mathrm{EAAAK})_{6} \mathrm{~A}(\mathrm{GSG})_{6}\right) .2 \mathrm{nM}$ sensor incubated with hCRP for 30 min before $50 \mu \mathrm{M}$ nitrocefin addition. Fold activity gain $=\Delta A_{551}$ $(\mathrm{hCRP}) / \Delta A_{551}(0 \mathrm{nM}$ hCRP) measured after a further $30 \mathrm{~min}$. (B) Optimizing L2 (with L1 = TSA, L3 = AAA). 2 nM sensor incubated with hCRP for $10 \mathrm{~min}$ before $50 \mu \mathrm{M}$ nitrocefin addition. Read after a further $10 \mathrm{~min}$ and displayed as raw $A_{551}$ to show background activity. (C) Reoptimizing L1 (with L2 $\left.=(\mathrm{GSG})_{7}, \mathrm{~L} 3=\mathrm{AAA}\right) .2 \mathrm{nM}$ sensor incubated with hCRP for 10 min before $50 \mu \mathrm{M}$ nitrocefin addition. Fold activity gain measured after a further $4 \mathrm{~min}$. (D) Dose response of optimized hCRP sensor BB hCRP $\left(\mathrm{BLA}^{-}{ }^{\mathrm{L} 1} \mathrm{TSA}^{\mathrm{A}} 90_{-}{ }^{\mathrm{L} 2}(\mathrm{GSG}) \mathrm{7}^{-}{ }^{\mathrm{B}} 90_{-}{ }^{\mathrm{L} 3} \mathrm{AAA}-\mathrm{BLIP}\right)$ and $^{-}$ controls where Aff-hCRP90 at site A or B is replaced with a non-specific Affimer, Aff-NS (BB_hCRP ${ }_{-}^{\mathrm{A}} \mathrm{Aff}-\mathrm{NS}, \mathrm{BB}$ hCRP ${ }^{\mathrm{B}} \mathrm{Aff}-\mathrm{NS}$ ). 2 nM sensor incubated with hCRP (or lysozyme as a control, where indicated) for $10 \mathrm{~min}$ before $50 \mu \overline{\mathrm{M}}$ nitrocefin addition. Fold activity gain read after a further $5 \mathrm{~min}$. Data are the mean of triplicates in six separate repeat experiments and error bars indicate standard deviation from the mean. The solid line is a $4 \mathrm{PL}$ regression fit and the dash line indicates the LoD.

(Figures $3 \mathrm{~A}$ and S3). ELISAs suggested that binding to hCRP was weaker at Affimer site A than B (Figure S4) but could be improved with a longer but rigid (helical or proline rich) L1 (Figure S5). However, there was no concomitant improvement in sensor response (Figure S6), suggesting L1 = TSA was not limiting.

The Aff-hCRP90 binding sites on hCRP are unknown, as is the orientation, distance, and pathway between them, so the SF linker 2 (L2) may not be optimal to enable Affimer binding in an orientation that stabilizes BLA-BLIP disruption. L2 was thus replaced by flexible glycine-serine linkers of different lengths (BLA- $\left.{ }^{\mathrm{L} 1} \mathrm{TSA}^{\mathrm{A}} 90^{\mathrm{L}}{ }^{\mathrm{2}}(\mathrm{GSG})_{n=5,7,20^{-}}{ }^{\mathrm{B}} 90^{-\mathrm{L} 3} \mathrm{AAA}-\mathrm{BLIP}\right)$, which greatly improved the sensor responses to hCRP (Figure $3 \mathrm{~B})$. With L2 $=(\mathrm{GSG})_{20}$, there was high background activity in the absence of hCRP, suggesting that a long flexible linker decreases the local BLIP concentration, thus reducing BLABLIP complex formation. A shorter L2 reduced background activity while $\mathrm{L} 2=(\mathrm{GSG})_{7}$ afforded the greatest activity at saturating hCRP concentrations, so this was used thereafter. ELISA results suggested that binding to hCRP was still slightly weaker at Affimer site A than B (Figure S7), so L1 was exchanged for the rigid linkers that previously enhanced binding at Affimer site A (BLA-L1- ${ }^{\mathrm{A}} 90^{-\mathrm{L} 2}$ (GSG) ${ }_{7}{ }^{\mathrm{B}} 90_{-}{ }^{\mathrm{L} 3} \mathrm{AAA}-$ BLIP with $\mathrm{L} 1=\mathrm{AP}$, APAP, APAPEPAP, $(\mathrm{APAPEP})_{2}$, $\left.(\text { EAAAK })_{2} \mathrm{G},(\text { EAAAK })_{3} \mathrm{P}\right)$. However, sensors with longer L1 had weakened dose responses to hCRP (Figure 3C). Despite the supposed rigidity of the longer helical or proline rich linkers, their length may allow BLA to remain bound to BLIP, even when the Affimer proteins bind hCRP. L1 = TSA was thus retained and the final sensor architecture was BLA- ${ }^{\mathrm{L} 1}$ TSA- ${ }^{\mathrm{A}} 90-{ }^{\mathrm{L} 2}$ (GSG) $)_{7^{-}}{ }^{\mathrm{B}} 90-{ }^{\mathrm{L} 3}$ AAA-BLIP (BB_hCRP). When a non-specific Affimer (Aff-NS) replaced Aff-hCRP90 at Affimer site $\mathrm{A}$ or $\mathrm{B}$ (BB_hCRP ${ }_{-}^{\mathrm{A}} \mathrm{Aff}-\mathrm{NS}, \mathrm{BB}$ hCRP ${ }_{-}^{\mathrm{B}} \mathrm{Aff}-$ NS), no dose response to hCRP was observed (Figure $3 \mathrm{D}$ ), confirming that BLA-BLIP disruption is only achieved when both Aff-hCRP90 Affimers bind to hCRP. Two key sensor design principles have emerged from engineering BB_hCRP. L2 should allow Affimer-target binding in an orientation that stabilizes the active conformation and L1/L3 should be short to ensure this disruption of BLA-BLIP.

The dose response of $\mathrm{BB}$ hCRP was tested in triplicate in six separate nitrocefin assays (Figure $3 \mathrm{D}$ ). At saturating hCRP concentrations $(30 \mathrm{nM})$, the sensor displayed a 12.6 fold activity gain from its background activity in the absence of hCRP. In fact, the activity of BB_hCRP in the presence of 30 nM hCRP was ca. $83 \%$ of TEM1- $\beta$-lactamase itself, showing that activity recovery has been well optimized and is much improved over some split enzyme systems, for example. ${ }^{11,13}$ The dose response curve can be fit to a $4 \mathrm{PL}$ regression with $C_{50}=0.5 \mathrm{nM}$ and the $\mathrm{LoD}=30 \mathrm{pM}$. Selectivity was also 
(A)

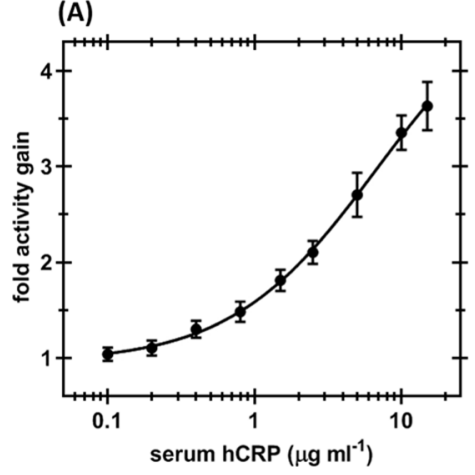

(B)

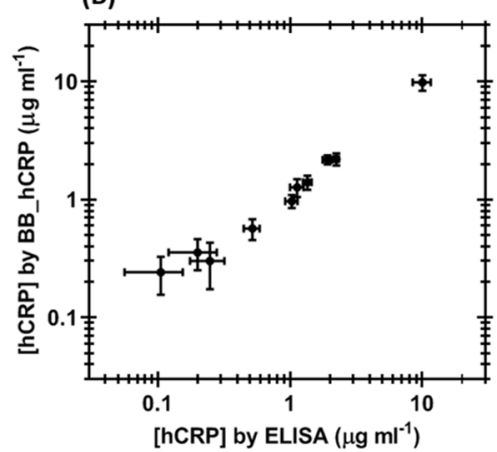

(c)

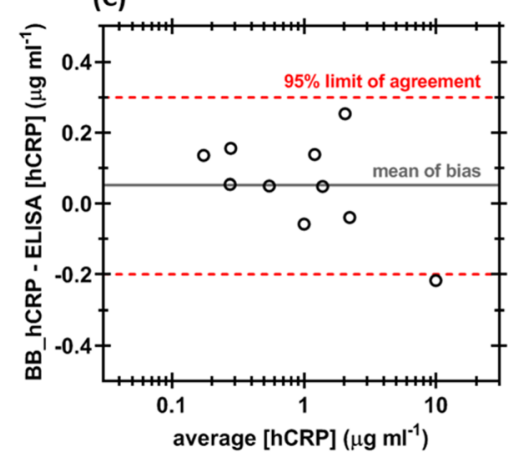

Figure 4. Quantifying hCRP in patient samples. (A) Calibration curve of BB_hCRP response to hCRP spiked in human serum. 2 nM BB_hCRP was incubated with hCRP spiked in hCRP-free serum (diluted 100 -fold) for $\overline{10}$ min before $50 \mu \mathrm{M}$ nitrocefin addition. $A_{486}$ and fold activity gain $=$ $\Delta A_{486}(\mathrm{hCRP}) / \Delta A_{486}(0 \mathrm{nM}$ hCRP $)$ were read after a further $10 \mathrm{~min}$. Data are the mean of eight independent measurements and error bars indicate standard deviation from the mean. The solid line is a $4 \mathrm{PL}$ regression fit. (B) Comparison of hCRP quantification in 10 patient samples by BB hCRP and ELISA. The BB hCRP assay was performed as for the calibration curve, with patient plasma diluted 100-fold; the hCRP concentration was interpolated from the standard calibration curve using the measured $A_{486}$ and fold activity gain. Data are the mean of eight independent measurements. CRP human ELISA kit (Invitrogen) was performed according to manufacturer instructions. Data are the mean of two independent sets of triplicates and error bars indicate standard deviation from the mean. (C) Bland-Altman analysis of hCRP quantification by BB_hCRP and ELISA.

observed, as BB_hCRP showed no activity recovery with nonspecific lysozyme protein as the target analyte. The maximal activity gain, $C_{50}$, and LoD of BB_hCRP are all improved over those measured for BB_HA and $\mathrm{BB}$ _Her, so redesign of the underlying enzyme-switch architecture has transformed an antibody sensor into a functional hCRP sensor.

Performance in Patient Samples. Serum samples were diluted 100-fold in the assay to achieve a sensor response in the range indicative of CVD risk (ca. $0.5-10 \mu \mathrm{g} \mathrm{mL}^{-1}=4.4-$ $87 \mathrm{nM}$ ); low, moderate, and high risk are indicated by $<1,1-3$, and $>3 \mu \mathrm{g} \mathrm{mL}{ }^{-1} \mathrm{hCRP}$, respectively. ${ }^{58} \mathrm{BB}$ hCRP displayed a comparable dose response in $1 \%$ human serum to that measured in buffer, so minimal matrix effects were observed (Figure S8). A calibration curve was established with nominal hCRP concentrations $\left(0.1-15 \mu \mathrm{g} \mathrm{mL}^{-1}\right)$ spiked into hCRPfree human serum in eight independent repeat experiments (Figure 4A). Samples were incubated with $2 \mathrm{nM} \mathrm{BB}$ hCRP for $10 \mathrm{~min}$, and $A_{486}$ was measured a further $10 \mathrm{~min}$ after $50 \mu \mathrm{M}$ nitrocefin addition. The BB hCRP and nitrocefin concentrations (Figure S9) and the incubation time (Figure S10) were optimized to balance speed with response gained, so must be adhered to. The optimal nitrocefin measurement wavelength $(486 \mathrm{~nm})$ was used to enhance sensitivity. The fold activity gain measurement $\left(\Delta A_{486}(\mathrm{hCRP}) / \Delta A_{486}(0 \mathrm{nM}\right.$ hCRP)) was used as it gives a more consistent response than raw $A_{486}$ (Figure S11). The zero hCRP reference adjusts for changes in activity due to external factors such as temperature. The dose response to serum hCRP was fit to a $4 \mathrm{PL}$ regression with $C_{50}=6.8 \mu \mathrm{g} \mathrm{mL}^{-1}(60 \mathrm{nM})$, so it compares well with the 100 -fold dilution measurement of $C_{50}=0.5 \mathrm{nM}$ observed in buffer. The LoD is $0.4 \mu \mathrm{g} \mathrm{mL} L^{-1}(3.5 \mathrm{nM})$ serum hCRP, equivalent to $35 \mathrm{pM}$ in the assay, and so is comparable to the $30 \mathrm{pM}$ LoD in buffer. For each individual measurement, the concentration was interpolated from the curve to assess the accuracy (by \% recovery) and precision (by \% coefficient of variation, $\mathrm{CV}$ ) of hCRP quantification in serum. Between 0.8 and $15 \mu \mathrm{g} \mathrm{mL}^{-1}$, the recovery $=96-106 \%$ and $\mathrm{CV} \leq 25 \%$, so the assay offers excellent accuracy and good precision over the clinically relevant range.
The BB_hCRP assay was then used to quantify hCRP in 10 patient samples, by interpolating the concentration from the standard calibration curve (Figure 4A). This was compared with a validated ELISA kit (CRP human ELISA kit, Invitrogen). Eight independent BB hCRP assays were performed and two independent ELISAs were carried out in triplicate. The measured hCRP concentrations were highly consistent between methods (Figure 4B) and a linear regression gave a slope of 0.97 with $R^{2}=0.999$. A BlandAltman analysis further assessed agreement between methods; for each patient sample, the difference between the two measures was plotted against their average. ${ }^{59}$ The mean of the bias between the two measurements was small $(0.053 \mu \mathrm{g}$ $\mathrm{mL}^{-1}$ ), and $95 \%$ of BB_hCRP measurements should fall within -0.2 to $+0.3 \mu \mathrm{g} \mathrm{mL}^{-1}$ of that measured by ELISA (Figure 4C). For samples with $>0.4 \mu \mathrm{g} \mathrm{mL}^{-1}$, the BB_hCRP assay gave 94$113 \%$ of the concentration measured by ELISA and CV $<20 \%$, thus confirming good agreement and reproducibility over the clinically relevant CVD prognosis range. For patient samples with $<0.4 \mu \mathrm{g} \mathrm{mL}^{-1} \mathrm{hCRP}$, agreement and reproducibility were understandably weaker, given the $0.4 \mu \mathrm{g} \mathrm{mL} \mathrm{m}^{-1}$ LoD. Nonetheless, for CVD prognosis, it is not important to quantify such low concentrations but just to determine they are $<1 \mu \mathrm{g} \mathrm{mL} L^{-1}$.

The BB_hCRP assay compares well with the ELISA but has numerous advantages for a point-of-care setting. The BB_hCRP assay requires only a $2 \mu \mathrm{L}$ sample, has no wash steps, and takes ca. $20 \mathrm{~min}$, whereas the ELISA requires multiple wash steps and takes hours. The ELISA also requires a full calibration curve with each assay, whereas the BB hCRP assay uses a standard calibration curve. The only requirement is that a supplied hCRP-free serum reference must be run in parallel, so the fold activity gain can be calculated. The BB_hCRP assay thus holds several advantages for a point-ofcare hCRP test for rapid screening of patients with elevated CVD risk. More broadly, the underlying enzyme-switch is proven to be capable of rapid, no-wash protein detection within a patient sample matrix, offering promise for development of sensors for further human biomarkers. 
(A)

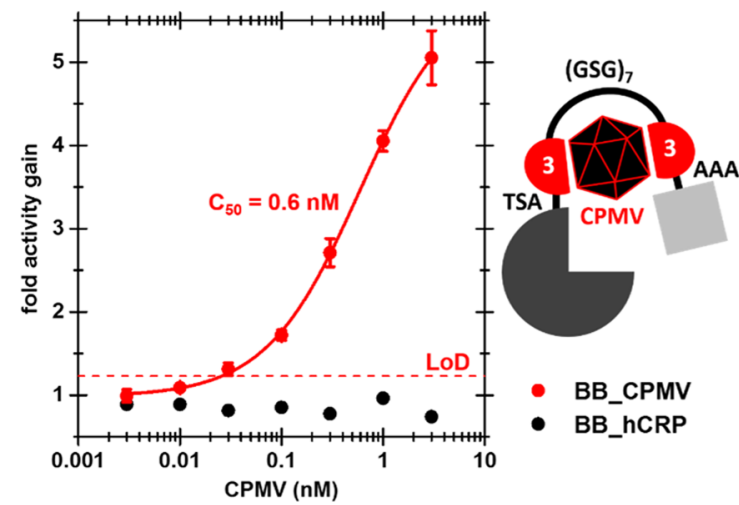

(B)

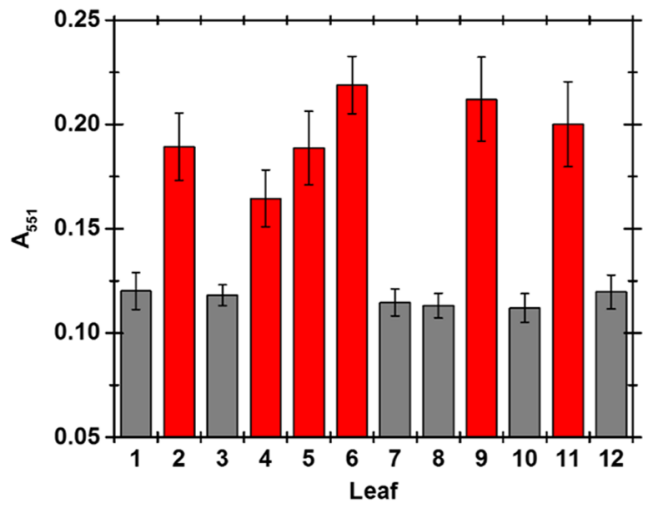

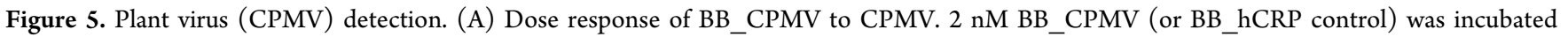

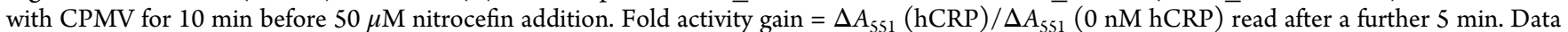
points are the mean of three independent measurements, and error bars indicate standard deviation from the mean. The solid line is a 4PL regression fit and the dash line indicates the LoD. (B) Detection of CPMV in infected leaves. Crude extracts of six healthy (gray) and six CPMV infected (red) leaves were prepared with the P-PER plant protein extraction kit (ThermoFisher), and 2 nM BB_CPMV was incubated with 20-fold diluted extract for $15 \mathrm{~min}$ before $50 \mu \mathrm{M}$ nitrocefin addition. $A_{551}$ read after a further $20 \mathrm{~min}$. Data are the mean of three independent measurements, and error bars indicate standard deviation from the mean.

Other biomarkers of interest are likely to be present at lower serum concentrations and so require the assay to work at higher serum concentrations. In 5\% human serum, BB hCRP is partly inhibited (Figure S8) but, when read after 10 min, the fold activity gain dose response is similar to that in buffer (Figure S12). Nitrocefin can break down in serum but with 5\% this occurs more slowly than the timescale of the assay (Figure S13). For measurements in higher percentages of serum, the serum-stable fluorescent substrate CCF2-FA can be used. ${ }^{24} \mathrm{~A}$ dose response was obtained in $10 \%$ serum, but the sensor response was again inhibited (Figure S14). BB_hCRP thus functions best in a $\leq 1 \%$ serum matrix; dose response curves are achievable in up to $10 \%$ serum, but further development is required to reduce matrix effects.

Batch-to-Batch Reproducibility and Stability. Batchto-batch reproducibility of reagents is important in ensuring consistent manufacture of point-of-care tests. Three separate batches of BB_hCRP were produced and purified; each had a very similar dose response to hCRP, in terms of both absolute response (Figure S15A) and fold activity gain (Figure S15B), with $C_{50}=0.3-0.5 \mathrm{nM}$.

The stability of BB_hCRP was assessed by comparing

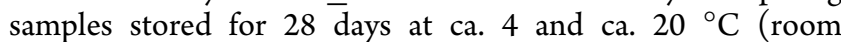
temperature) with those from freezer storage at $-80{ }^{\circ} \mathrm{C}$. Room temperature storage for 28 days reduced the absolute response of BB_hCRP, by ca. $11 \%$ with $10 \mathrm{nM}$ hCRP (Figure S15C). Importantly, the dose response in terms of fold activity gain was very similar regardless of whether samples were freshly thawed or stored at 4 or $20^{\circ} \mathrm{C}$ (Figure S15D), with $C_{50}=0.3-$ $0.5 \mathrm{nM}$. This confirms the robustness of BB_hCRP to longterm storage, even at room temperature, which is important for simple storage and handling requirements of point-of-care tests.

Example 3: Detection of Plant Virus (CPMV) Infection in Leaf Samples. CPMV is a viral plant pathogen that causes severe crop loss in cowpea. It is one of a number of plant pathogens for which rapid in-field diagnostics would improve early disease detection, allowing containment to avoid crop losses. ${ }^{4,47,60}$ To generate a CPMV sensor, each Affimer in BB_hCRP was exchanged for an Aff-CPMV3 Affimer (3). The rest of the sensor architecture was retained to give
BLA- ${ }^{\mathrm{L} 1} \mathrm{TSA}^{\mathrm{A}}{ }_{3}{ }^{-\mathrm{L} 2}(\mathrm{GSG}){ }_{7}{ }^{-}{ }^{\mathrm{B}}{ }_{-}{ }^{\mathrm{L} 3} \mathrm{AAA}-\mathrm{BLIP}$ (BB_CPMV). In the nitrocefin assay $B B_{-} C P M V$, but not nonspecific BB hCRP, was activated by CPMV and showed a maximum ca. 5-fold activity gain. The dose response fit to a $4 \mathrm{PL}$ regression with $C_{50}=0.6 \mathrm{nM}$ and the $\mathrm{LoD}=30 \mathrm{pM}$ (Figure $5 A)$. These values are comparable to those obtained for BB_hCRP with hCRP, showing that under these conditions, the underlying enzyme-switch structure provides similar functionality. However, it should be noted that $C_{50}$ is measured using $2 \mathrm{nM}$ sensor and differences in effective sensor $K_{\mathrm{d}}$ may be revealed by measurements of initial rates at lower sensor concentrations. The sensor $C_{50}$ values, as measured, are lower than the respective Affimer $K_{\mathrm{d}}$ 's; Banala et al. have previously shown that a bivalent interaction by a single protein switch enhances sensitivity by an avidity effect and offers a potential advantage over two component systems. ${ }^{24}$

BB CPMV was tested for its ability to identify CPMVinfected leaves in a blind sample assay. Crude extracts of six healthy and six CPMV-infected leaves were prepared using the P-PER plant protein extraction kit (ThermoFisher), according to the manufacturer's instructions. These samples, labeled 112 , were then provided for assay and BB_CPMV ( $2 \mathrm{nM})$ was incubated with a 20-fold diluted aliquot of leaf extract for 15 min, before addition of $50 \mu \mathrm{M}$ nitrocefin. $A_{551}$ was read after a further $20 \mathrm{~min}$ (Figure 5B). All infected leaf samples gave a statistically higher absorbance value than healthy leaves $(p<$ 0.001). This demonstrates the selectivity of the sensor, as it is able to detect CPMV within the crude leaf extract matrix. The assay is simple and takes ca. $45 \mathrm{~min}$, including a 10 min leaf preparation and so shows suitability for rapid in-field diagnostics. CPMV is one of many pathogens for which such diagnostics would significantly improve crop disease management.

The generation of a functional sensor for an icosahedral virus from one for a pentameric protein, hCRP, by simple exchange of Affimer proteins suggests that the sensor architecture can be used for different multimeric targets. This is further supported by the generation of a functional Herceptin antibody sensor via exchange of Aff-Her Affimer proteins into the same sensor structure (BLA- ${ }^{\mathrm{L} 1} \mathrm{TSA}-{ }^{\mathrm{A}} \mathrm{Aff}-$ Her- $^{\mathrm{L} 2}(\mathrm{GSG})_{7^{-}}{ }^{\mathrm{B}}$ Aff-Her- ${ }^{\mathrm{L} 3}$ AAA-BLIP) (Figure S16). Such 
modularity is vital if a sensor is to be used as a general platform for point-of-care or in-field assays. Broader multimeric protein detection was achieved through simple exchange of rapidly selected Affimer proteins capable of binding at two repeated binding sites on the analyte. ${ }^{32}$ However, different targets might have different architectural constraints with different optimal linkers. Further widening the target scope to proteins without repeated binding sites would require selection of Affimers against two non-overlapping epitopes and potentially further sensor development. It seems likely that in the present configuration, there will be a lower limit to the size of the protein that can be detected because of (a) the increased difficulty of identifying binders that recognize two nonoverlapping sites in small proteins and (b) that such sites may not be sufficiently distant to provide the conformational disruption for dissociation of BLA and BLIP in the sensor.

\section{CONCLUSIONS}

We have engineered an enzyme-inhibitor switch sensor capable of rapid, picomolar quantification of multimeric protein targets in biological samples, using a simple, washfree assay format. This was achieved by incorporating nonimmunoglobulin Affimer binding proteins together with linker sequences between TEM1- $\beta$-lactamase (BLA) and its inhibitor protein (BLIP), such that target binding disrupts the enzymeinhibitor complex, switching on enzymatic activity. Nonimmunoglobulin binding proteins are highly successful antibody alternatives that can be rapidly selected in vitro against diverse targets. The enzyme-inhibitor switch provides a modular system to transform such passive binders into "active" signal generating sensors. ${ }^{61}$ A sensor for the therapeutic antibody Herceptin was generated by replacing epitope sequences with Herceptin-binding Affimer proteins in a previously reported antibody sensor structure. ${ }^{24}$ Two key design principles were required to engineer a more general enzyme-switch architecture for broader multimeric protein detection. First, the linker between the Affimers must be able to bridge the unknown pathway between binding sites on the analyte protein and allow Affimer-target binding in an orientation that stabilizes the active conformation. Second, the linkers between BLA-Affimer A and Affimer B-BLIP need to be short to ensure this BLA-BLIP disruption upon target binding.

Sensors for three target classes, a protein biomarker hCRP, a plant virus CPMV, and a therapeutic antibody Herceptin, were then generated by exchanging only the Affimer proteins in the basic enzyme-switch structure. This confirms a degree of modularity for the sensor against multimeric targets, which is essential for simple and timely development of sensors. The linkers do offer regions for sensor fine tuning, as different targets might have different architectural constraints with different optimal linkers. The enzyme-inhibitor switch affords a wider protein target scope than reported BRET-based sensors $^{14-20}$ and provides enhanced activity recovery and stability compared to split-enzyme complementation approaches. ${ }^{11-13}$ A recently reported two-component allosteric enzyme system offers an improved dynamic response, but the single-protein enzyme-inhibitor switch reported here provides avidity for enhanced sensitivity and circumvents self-association that can cause a performance-limiting sensor concentration dependence. ${ }^{23}$ The enzyme-inhibitor switch offers simplicity, speed, stability, and batch-to-batch reproducibility, with potential advantages for clinical point-of-care testing and in-field diagnostics, exemplified by relevant assays for therapeutic dose monitoring, health diagnostics, and plantpathogen detection. Integration into a portable diagnostic device may require development of an amperometric assay exploiting the electrochemical activity of hydrolyzed nitrocefin, to avoid optical detection and aid miniaturisation, ${ }^{62}$ or identification of an improved enzyme system affording a calibration-free ratiometric response and reduced serum interference. Auto-inhibited protein switches are used in Nature for sensing and regulation, and it is hoped that the general principles learned here can aid their translation into essential tools for synthetic biology, molecular imaging, and high-throughput screening, in addition to their molecular diagnostic applications.

\section{EXPERIMENTAL SECTION}

Detailed methods for Affimer selection and validation, SPR and sensor cloning, mutagenesis, expression, purification and characterization are provided in the Supporting Information.

\section{ASSOCIATED CONTENT}

\section{S Supporting Information}

The Supporting Information is available free of charge on the ACS Publications website at DOI: 10.1021/acssensors.9b01574.

Experimental section, supplementary figures, DNA and protein sequences, and tables of primers (PDF)

\section{AUTHOR INFORMATION}

\section{Corresponding Authors}

*E-mail: D.C.Tomlinson@leeds.ac.uk (D.C.T.).

*E-mail: L.J.C.Jeuken@leeds.ac.uk (L.J.C.J.).

ORCID $\odot$

Hope Adamson: 0000-0002-2582-3287

Anna A. Tang: 0000-0002-5216-5080

Michael J. McPherson: 0000-0002-0719-6427

Lars J. C. Jeuken: 0000-0001-7810-3964

\section{Notes}

The authors declare the following competing financial interest(s): MJM and DCT are named inventors on granted patent US 9,932,575 owned by the University of Leeds and licensed to Avacta Life Sciences Ltd, and own some personal shares in Avacta Life Sciences. RF, AD and MJ all work for Avacta Life Sciences.

\section{ACKNOWLEDGMENTS}

We gratefully thank Emma Hesketh, Neil Ranson and George Lomonossoff and his group for their input and provision of purified CPMV and CPMV infected leaf samples. This work was funded by the Medical Research Council, MRC (MR/ N029976/1).

\section{REFERENCES}

(1) Nayak, S.; Blumenfeld, N. R.; Laksanasopin, T.; Sia, S. K. Pointof-care diagnostics: Recent developments in a connected age. Anal. Chem. 2017, 89, 102-123.

(2) Jani, I. V.; Peter, T. F. How point-of-care testing could drive innovation in global health. N. Engl. J. Med. 2013, 368, 2319-2324.

(3) Knopp, D. Immunoassay development for environmental analysis. Anal. Bioanal. Chem. 2006, 385, 425-427. 
(4) Cassedy, A.; Mullins, E.; O'Kennedy, R. Sowing seeds for the future: The need for on-site plant diagnostics. Biotechnol. Adv. 2019, DOI: $10.1016 /$ j.biotechadv.2019.02.014.

(5) Lequin, R. M. Enzyme immunoassay (EIA)/enzyme-linked immunosorbent assay (ELISA). Clin. Chem. 2005, 51, 2415-2418.

(6) Engvall, E.; Perlmann, P. Enzyme-linked immunosorbent assay (ELISA) quantitative assay of immunoglobulin G. Immunochemistry 1971, 8, 871-874.

(7) Vallée-Bélisle, A.; Plaxco, K. W. Structure-switching biosensors: inspired by Nature. Curr. Opin. Struct. Biol. 2010, 20, 518-526.

(8) Jung, W.; Han, J.; Choi, J.-W.; Ahn, C. H. Point-of-care testing (POCT) diagnostic systems using microfluidic lab-on-a-chip technologies. Microelectron. Eng. 2015, 132, 46-57.

(9) Koczula, K. M.; Gallotta, A. Lateral flow assays. Essays Biochem. 2016, 60, 111-120.

(10) Stein, V.; Alexandrov, K. Synthetic protein switches: design principles and applications. Trends Biotechnol. 2015, 33, 101-110.

(11) Guo, Z.; Murphy, L.; Stein, V.; Johnston, W. A.; Alcala-Perez, S.; Alexandrov, K. Engineered PQQ-glucose dehydrogenase as a universal biosensor platform. J. Am. Chem. Soc. 2016, 138, 1010810111.

(12) Dixon, A. S.; Kim, S. J.; Baumgartner, B. K.; Krippner, S.; Owen, S. C. A Tri-part Protein Complementation System Using Antibody-Small Peptide Fusions Enables Homogeneous Immunoassays. Sci. Rep. 2017, 7, 8186.

(13) Su, J.; Dong, J.; Kitaguchi, T.; Ohmuro-Matsuyama, Y.; Ueda, $\mathrm{H}$. Noncompetitive homogeneous immunodetection of small molecules based on beta-glucuronidase complementation. Analyst 2018, 143, 2096-2101.

(14) Yu, Q.; Xue, L.; Hiblot, J.; Griss, R.; Fabritz, S.; Roux, C.; Binz, P.-A.; Haas, D.; Okun, J. G.; Johnsson, K. Semisynthetic sensor proteins enable metabolic assays at the point of care. Science 2018, $361,1122-1126$.

(15) Xue, L.; Yu, Q.; Griss, R.; Schena, A.; Johnsson, K. Bioluminescent Antibodies for Point-of-Care Diagnostics. Angew. Chem. 2017, 129, 7218-7222.

(16) Griss, R.; Schena, A.; Reymond, L.; Patiny, L.; Werner, D.; Tinberg, C. E.; Baker, D.; Johnsson, K. Bioluminescent sensor proteins for point-of-care therapeutic drug monitoring. Nat. Chem. Biol. 2014, 10, 598-603.

(17) Tenda, K.; van Gerven, B.; Arts, R.; Hiruta, Y.; Merkx, M.; Citterio, D. Paper-Based Antibody Detection Devices Using Bioluminescent BRET-Switching Sensor Proteins. Angew. Chem. 2018, 130, 15595-15599.

(18) Arts, R.; Ludwig, S. K. J.; van Gerven, B. C. B.; Estirado, E. M.; Milroy, L.-G.; Merkx, M. Semisynthetic bioluminescent sensor proteins for direct detection of antibodies and small molecules in solution. ACS Sens. 2017, 2, 1730-1736.

(19) Schena, A.; Griss, R.; Johnsson, K. Modulating protein activity using tethered ligands with mutually exclusive binding sites. Nat. Commun. 2015, 6, 7830.

(20) Dale, N. C.; Johnstone, E. K. M.; White, C.; Pfleger, K. D. NanoBRET: The Bright Future of Proximity-Based Assays. Front. Bioeng. Biotechnol. 2019, 7, 56.

(21) Nicholes, N.; Date, A.; Beaujean, P.; Hauk, P.; Kanwar, M.; Ostermeier, M. Modular protein switches derived from antibody mimetic proteins. Protein Eng., Des. Sel. 2015, 29, 77-85.

(22) Tullman, J.; Nicholes, N.; Dumont, M. R.; Ribeiro, L. F.; Ostermeier, M. Enzymatic protein switches built from paralogous input domains. Biotechnol. Bioeng. 2016, 113, 852-858.

(23) Guo, Z.; Johnston, W. A.; Whitfield, J.; Walden, P.; Cui, Z.; Wijker, E.; Edwardraja, S.; Retamal Lantadilla, I.; Ely, F.; Vickers, C.; et al. Generalizable Protein Biosensors Based on Synthetic Switch Modules. J. Am. Chem. Soc. 2019, 141, 8128-8135.

(24) Banala, S.; Aper, S. J. A.; Schalk, W.; Merkx, M. Switchable reporter enzymes based on mutually exclusive domain interactions allow antibody detection directly in solution. ACS Chem. Biol. 2013, 8, 2127-2132.
(25) de las Heras, R.; Fry, S. R.; Li, J.; Arel, E.; Kachab, E. H.; Hazell, S. L.; Huang, C.-Y. Development of homogeneous immunoassays based on protein fragment complementation. Biochem. Biophys. Res. Commun. 2008, 370, 164-168.

(26) Banala, S.; Arts, R.; Aper, S. J. A.; Merkx, M. No washing, less waiting: engineering biomolecular reporters for single-step antibody detection in solution. Org. Biomol. Chem. 2013, 11, 7642-7649.

(27) Stein, V.; Alexandrov, K. Protease-based synthetic sensing and signal amplification. Proc. Natl. Acad. Sci. U.S.A. 2014, 111, 1593415939.

(28) Stein, V.; Nabi, M.; Alexandrov, K. Ultrasensitive scaffolddependent protease sensors with large dynamic range. ACS Synth. Biol. 2017, 6, 1337-1342.

(29) Nirantar, S. R.; Yeo, K. S.; Chee, S.; Lane, D. P.; Ghadessy, F. J. A generic scaffold for conversion of peptide ligands into homogenous biosensors. Biosens. Bioelectron. 2013, 47, 421-428.

(30) Kim, H.; Yoon, H. K.; Yoo, T. H. Engineering $\beta$-lactamase zymogens for use in protease activity assays. Chem. Commun. 2014, 50, 10155-10157.

(31) Škrlec, K.; Štrukelj, B.; Berlec, A. Non-immunoglobulin scaffolds: a focus on their targets. Trends Biotechnol. 2015, 33, 408418.

(32) Tiede, C.; Bedford, R.; Heseltine, S. J.; Smith, G.; Wijetunga, I.; Ross, R.; AlQallaf, D.; Roberts, A. P.; Balls, A.; Curd, A.; et al. Affimer proteins are versatile and renewable affinity reagents. eLife 2017, 6, No. e24903.

(33) Tiede, C.; Tang, A. A. S.; Deacon, S. E.; Mandal, U.; Nettleship, J. E.; Owen, R. L.; George, S. E.; Harrison, D. J.; Owens, R. J.; Tomlinson, D. C.; et al. Adhiron: a stable and versatile peptide display scaffold for molecular recognition applications. Protein Eng., Des. Sel. 2014, 27, 145-155.

(34) Tang, A. A.-S.; Tiede, C.; Hughes, D. J.; McPherson, M. J.; Tomlinson, D. C. Isolation of isoform-specific binding proteins (Affimers) by phage display using negative selection. Sci. Signaling 2017, 10, No. eaan0868.

(35) Zhurauski, P.; Arya, S. K.; Jolly, P.; Tiede, C.; Tomlinson, D. C.; Ko Ferrigno, P.; Estrela, P. Sensitive and selective Affimerfunctionalised interdigitated electrode-based capacitive biosensor for Her4 protein tumour biomarker detection. Biosens. Bioelectron. 2018, $108,1-8$.

(36) Martin, H. L.; Bedford, R.; Heseltine, S. J.; Tang, A. A.; Haza, K. Z.; Rao, A.; McPherson, M. J.; Tomlinson, D. C. Nonimmunoglobulin scaffold proteins: Precision tools for studying protein-protein interactions in cancer. New Biotechnol. 2018, 45, $28-35$.

(37) Xie, C.; Tiede, C.; Zhang, X.; Wang, C.; Li, Z.; Xu, X.; McPherson, M. J.; Tomlinson, D. C.; Xu, W. Development of an Affimer-antibody combined immunological diagnosis kit for glypican3. Sci. Rep. 2017, 7, 9608.

(38) Wang, W.; Guo, Y.; Tiede, C.; Chen, S.; Kopytynski, M.; Kong, Y.; Kulak, A.; Tomlinson, D.; Chen, R.; McPherson, M.; et al. Ultraefficient cap-exchange protocol to compact biofunctional quantum dots for sensitive ratiometric biosensing and cell imaging. ACS Appl. Mater. Interfaces 2017, 9, 15232-15244.

(39) Koutsoumpeli, E.; Tiede, C.; Murray, J.; Tang, A.; Bon, R. S.; Tomlinson, D. C.; Johnson, S. Antibody mimetics for the detection of small organic compounds using a quartz crystal microbalance. Anal. Chem. 2017, 89, 3051-3058.

(40) Sharma, R.; Deacon, S. E.; Nowak, D.; George, S. E.; Szymonik, M. P.; Tang, A. A. S.; Tomlinson, D. C.; Davies, A. G.; McPherson, M. J.; Wälti, C. Label-free electrochemical impedance biosensor to detect human interleukin- 8 in serum with sub-pg/ml sensitivity. Biosens. Bioelectron. 2016, 80, 607-613.

(41) Hughes, D. J.; Tiede, C.; Penswick, N.; Tang, A. A.-S.; Trinh, C. H.; Mandal, U.; Zajac, K. Z.; Gaule, T.; Howell, G.; Edwards, T. A.; et al. Generation of specific inhibitors of SUMO-1-and SUMO-2/ 3-mediated protein-protein interactions using Affimer (Adhiron) technology. Sci. Signaling 2017, 10, No. eaaj2005. 
(42) Lopata, A.; Hughes, R.; Tiede, C.; Heissler, S. M.; Sellers, J. R.; Knight, P. J.; Tomlinson, D.; Peckham, M. Affimer proteins for Factin: novel affinity reagents that label F-actin in live and fixed cells. Sci. Rep. 2018, 8, 6572.

(43) Schlichthaerle, T.; Eklund, A. S.; Schueder, F.; Strauss, M. T.; Tiede, C.; Curd, A.; Ries, J.; Peckham, M.; Tomlinson, D. C.; Jungmann, R. Site-specific labeling of affimers for DNA-PAINT microscopy. Angew. Chem., Int. Ed. 2018, 57, 11060-11063.

(44) Nahta, R.; Esteva, F. J. Herceptin: mechanisms of action and resistance. Cancer Lett. 2006, 232, 123-138.

(45) Mould, D. Why therapeutic drug monitoring is needed for monoclonal antibodies and how do we implement this? Clin. Pharmacol. Ther. 2016, 99, 351-354.

(46) Vashist, S. K.; Venkatesh, A. G.; Marion Schneider, E.; Beaudoin, C.; Luppa, P. B.; Luong, J. H. T. Bioanalytical advances in assays for C-reactive protein. Biotechnol. Adv. 2016, 34, 272-290.

(47) Hesketh, E. L.; Tiede, C.; Adamson, H.; Adams, T. L.; Byrne, M. J.; Meshcheriakova, Y.; Kruse, I.; McPherson, M. J.; Lomonossoff, G. P.; Tomlinson, D. C.; et al. Affimer reagents as tools in diagnosing plant virus diseases. Sci. Rep. 2019, 9, 7524.

(48) Adamson, H.; Nicholl, A.; Tiede, C.; Tang, A. A.; Davidson, A.; Curd, H.; Wignall, A.; Ford, R.; Nuttall, J.; McPherson, M. J.; Johnson, M.; Tomlinson, D. C. Affimers as anti-idiotypic affinity reagents for pharmacokinetic analysis of biotherapeutics. BioTechniques 2019, in press DOI: 10.2144/btn-2019-0089

(49) Bruno, R.; Washington, C. B.; Lu, J.-F.; Lieberman, G.; Banken, L.; Klein, P. Population pharmacokinetics of trastuzumab in patients with HER2+ metastatic breast cancer. Cancer Chemother. Pharmacol. 2005, 56, 361-369.

(50) Armbruster, D. A.; Pry, T. Limit of blank, limit of detection and limit of quantitation. Clin. Biochem. Rev. 2008, 29, S49-S52.

(51) Leyland-Jones, B.; Colomer, R.; Trudeau, M. E.; Wardley, A.; Latreille, J.; Cameron, D.; Cubedo, R.; Al-Sakaff, N.; Feyereislova, A.; Catalani, O.; et al. Intensive Loading Dose of Trastuzumab Achieves Higher-Than-Steady-State Serum Concentrations and Is Well Tolerated. J. Clin. Oncol. 2009, 28, 960-966.

(52) van Rosmalen, M.; Ni, Y.; Vervoort, D. F. M.; Arts, R.; Ludwig, S. K. J.; Merkx, M. Dual-color bioluminescent sensor proteins for therapeutic drug monitoring of antitumor antibodies. Anal. Chem. 2018, 90, 3592-3599.

(53) Porchetta, A.; Ippodrino, R.; Marini, B.; Caruso, A.; Caccuri, F.; Ricci, F. Programmable nucleic acid nanoswitches for the rapid, single-step detection of antibodies in bodily fluids. J. Am. Chem. Soc. 2018, 140, 947-953.

(54) Clos, T. W. C. Pentraxins: Structure, Function, and Role in Inflammation. ISRN Inflammation 2013, 2013, 379040.

(55) Cooke, J.; Butler, C.; Hopstaken, R.; Dryden, M. S.; McNulty, C.; Hurding, S.; Moore, M.; Livermore, D. M. Narrative review of primary care point-of-care testing (POCT) and antibacterial use in respiratory tract infection (RTI). BMJ Open Respir. Res. 2015, 2, No. e000086.

(56) Ji, T.; Liu, D.; Liu, F.; Li, J.; Ruan, Q.; Song, Y.; Tian, T.; Zhu, Z.; Zhou, L.; Lin, H.; et al. A pressure-based bioassay for the rapid, portable and quantitative detection of C-reactive protein. Chem. Commun. 2016, 52, 8452-8454.

(57) Shrive, A. K.; Gheetham, G. M. T.; Holden, D.; Myles, D. A. A.; Turnell, W. G.; Volanakis, J. E.; Pepys, M. B.; Bloomer, A. C.; Greenhough, T. J. Three dimensional structure of human C-reactive protein. Nat. Struct. Biol. 1996, 3, 346-354.

(58) Ridker, P. M. Clinical application of C-reactive protein for cardiovascular disease detection and prevention. Circulation 2003, 107, 363-369.

(59) Giavarina, D. Understanding bland altman analysis. Biochem. Med. 2015, 25, 141-151.

(60) Khater, M.; de la Escosura-Muñiz, A.; Merkoçi, A. Biosensors for plant pathogen detection. Biosens. Bioelectron. 2017, 93, 72-86.

(61) Léger, C.; Di Meo, T.; Aumont-Nicaise, M.; Velours, C.; Durand, D.; de la Sierra-Gallay, I. L.; van Tilbeurgh, H.; Hildebrandt, N.; Desmadril, M.; Urvoas, A.; et al. Ligand-induced conformational switch in an artificial bidomain protein scaffold. Sci. Rep. 2019, 9, 1178.

(62) Rochelet, M.; Solanas, S.; Betelli, L.; Neuwirth, C.; Vienney, F.; Hartmann, A. Amperometric detection of extended-spectrum $\beta$ lactamase activity: application to the characterization of resistant $\mathrm{E}$. coli strains. Analyst 2015, 140, 3551-3556. 